\title{
Short-term outcomes of tonsillectomy in adult patients with recurrent pharyngitis: a randomized controlled trial
}

\author{
Timo Koskenkorva MD, Petri Koivunen MD PhD, Markku Koskela MD PhD, Onni Niemela MD PhD, \\ Aila Kristo MD PhD, Olli-Pekka Alho MD PhD
}

\begin{abstract}
Background: Limited evidence exists as to the benefit of tonsillectomy in adult patients. We sought to determine the short-term efficacy of tonsillectomy for recurrent pharyngitis in adults.

Methods: We conducted a randomized, controlled, parallel-group trial at a tertiary care ear, throat and nose centre in Oulu, Finland, between October 2007 and December 2010. Adult patients with recurrent pharyngitis were randomly assigned to the control group (watchful waiting) or the tonsillectomy group. Our primary outcome was the difference in the proportion of patients with severe pharyngitis (severe symptoms and C-reactive protein level $>40 \mathrm{mg} / \mathrm{L}$ ) within 5 months. Our secondary outcomes included differences between groups in proportions of patients who had episodes of pharyngitis with or without medical consultation, rates of pharyngitis and numbers of days with symptoms.
\end{abstract}

Results: Of 260 patients referred for tonsillectomy because of recurrent pharyngitis, we recruited 86 participants for our study. Of these, 40 patients were randomly allocated to the control group, and 46 were randomly allo- cated to the tonsillectomy group. One patient in the control group and no patients in the tonsillectomy group had a severe episode of pharyngitis (difference 3\%, 95\% confidence interval $[\mathrm{Cl}]-2 \%$ to $7 \%$ ). Seventeen patients in the control group (43\%) and 2 patients in the tonsillectomy group (4\%) consulted a physician for pharyngitis (difference $38 \%, 95 \% \mathrm{Cl}$ $22 \%$ to $55 \%$ ). Overall, 32 patients in the control group $(80 \%)$ and 18 patients in the tonsillectomy group $(39 \%)$ had an episode of pharyngitis during the 5-month follow-up (difference $41 \%, 95 \% \mathrm{Cl} 22 \%$ to $60 \%$ ). The rate of pharyngitis and number of symptomatic days were significantly lower in the tonsillectomy group than in the control group.

Interpretation: There was no significant difference in the number of episodes of severe pharyngitis between the control and treatment groups, and episodes were rare. However, tonsillectomy resulted in fewer symptoms of pharyngitis, consequently decreasing the number of medical visits and days absent from school or work. For this reason, surgery may benefit some patients. Trial registration: ClinicalTrials.gov, no. NCT00547391.
Competing interests: None declared.

This article has been peer reviewed.

Correspondence to: Timo Koskenkorva, timo.koskenkorva@ppshp.fi

CMAJ 2013. DOI:10.1503 /cmaj.121852
$\mathrm{R}$ ecurrent pharyngitis is a common health problem causing repeated use of antibiotic agents and absences from school or work. The condition has often been treated with tonsillectomy. ${ }^{1}$ According to a recent Cochrane review, tonsillectomy or adenotonsillectomy reduces the number of episodes and days with sore throat in children. ${ }^{2}$ However, the same review found limited evidence of benefit of tonsillectomy in adults.

Alho and colleagues have previously shown that adults with severe recurrent streptococcal pharyngitis received substantial benefit from tonsillectomy over a short period. ${ }^{3}$ However, some guidelines suggest that throat swabs should not be done routinely in primary care management of acute pharyngitis ${ }^{4,5}$ raising the question of how to treat recurrent episodes of pharyngitis when the cause is unknown. Thus, we sought to determine the effectiveness of tonsillectomy in reducing the number of episodes of severe pharyngitis among adult patients with recurrent pharyngitis of any origin.

\section{Methods}

\section{Study design}

We conducted a randomized, controlled, parallelgroup trial in a tertiary care ear, nose and throat centre. All patients provided written informed consent. The study protocol was approved by the Oulu University Hospital ethics committee.

\section{Participants}

We selected participants from consecutive patients referred for tonsillectomy because of recurrent pharyngitis from Oct. 29, 2007, to June 30, 2010. 
The clinical criterion for entry to the study was 3 or more episodes of pharyngitis within the previous 12 months. These episodes had to be disabling, prevent normal functioning, be severe enough for the patient to seek medical attention and be thought to involve the palatine tonsils. It was not necessary for culture or antigen tests to have shown infection with group A streptococcus. Our exclusion criteria were age less than 13 years, history of peritonsillar abscess, chronic tonsillitis, ongoing use of antibiotic agents, residence outside of the Oulu region, pregnancy or previous illness making same-day surgery unfeasible.

\section{Intervention}

We assigned patients to the control group or the tonsillectomy group using simple randomization. The allocation sequence was concealed from the investigators using sequentially numbered, opaque, sealed envelopes (Appendix 1, available at www.cmaj.ca/lookup/suppl/doi:10.1503/cmaj $.121852 /-/ \mathrm{DC} 1)$.

The patients in the control group were placed on a waiting list for tonsillectomy to undergo surgery after 5 to 6 months (watchful waiting); patients in the tonsillectomy group underwent surgery as soon as possible. Surgery involved total extracapsular removal of both palatine tonsils under general anesthesia. For practical reasons, the median time between the randomization to the tonsillectomy group and surgery was 14 (interquartile range 8-23) days.

\section{Study protocol}

Upon assignment to one of the study groups, patients underwent examination, and we collected background data. Both groups were scheduled to be followed for at least 5 months after randomization.

We advised the patients to visit the study physician or their general practitioner whenever they had acute symptoms suggestive of pharyngitis. In addition, we told patients that it was important to seek medical advice for their symptoms during the trial exactly as they had done before. At the acute visit, patients underwent a thorough clinical examination including a throat $\mathrm{swab}^{6}$ and a blood test to measure serum levels of C-reactive protein (Appendix 1). The blood test was repeated 3 days later. All laboratory and microbiological analyses were performed by staff blinded to the clinical data.

A study notebook provided to the patients included information about the study and written instructions for their general practitioners, which included information on examining and recording ear, throat and nose status and taking blood samples and throat cultures (Appendix 1). Patients received treatment as prescribed by a physician (the study physician if available), who recorded the date, location, diagnosis and treatment of acute episodes in the notebook. For patients in the tonsillectomy group, the study notebook also included a Glasgow Benefit Inventory healthrelated quality-of-life questionnaire ${ }^{7}$ to be answered 6 months after surgery. This instrument has been validated in Finnish by translation, reconciliation, back-translation and pilot testing. ${ }^{8}$

The patients used a symptom diary to record the presence and severity (mild, moderate or severe) of the following acute symptoms: throat pain, cough, rhinitis, fever and absence from school or work. Symptoms lasting more than 30 days were considered chronic and were not included in our analysis.

We collected the study notebooks at the followup visit. We checked missing or unreadable information by telephone. We recorded data concerning acute visits and tonsillectomy from patients' charts.

\section{Outcomes}

Our primary outcome was the difference in the proportion of patients who had a severe episode of pharyngitis within 5 months. A severe episode had to involve medical consultation registered in the study notebook, and the patient needed to have acute throat pain and signs suggesting the symptoms originated in the pharynx (e.g., edema, erythema, exudative tonsillitis, anterior cervical lymphadenitis). In addition, the serum level of C-reactive protein either on the day of the appointment or 3 days later had to be higher than $40 \mathrm{mg} / \mathrm{L} .{ }^{9}$ If a blood sample was not taken, the result of a throat culture had to show other than normal flora, and the patient had to grade the throat pain as severe. Secondary outcomes were differences in proportions of patients with any episode of pharyngitis (sore throat lasting $\geq 2 \mathrm{~d}$ ) and episodes with medical consultation during the 5-month follow-up, times to pharyngitis episodes, the difference in the mean rates of episodes, the mean number of days absent from school or work and the mean number of symptomatic days during follow-up. We also recorded health-related quality of life and adverse effects related to tonsillectomy.

\section{Statistical analysis}

We estimated that 70 patients needed to be enrolled in the study for it to have statistical power of $80 \%$ to detect an absolute difference of $25 \%$ in the recurrence rates of severe pharyngitis. We determined this estimate using a 5-month recurrence rate of $25 \%$ in the control group and $0 \%$ in the tonsillectomy group based on the results of the previous trial by Alho and col- 
leagues. ${ }^{3}$ We considered a 2 -sided $p$ value of 0.05 to be significant. We analyzed all of the participants on an intention-to-treat basis according to a pre-established plan.

For descriptive data, we calculated means with standard deviations or medians with interquartile ranges. We used the Mann-Whitney $U$ test to compare continuous variables. We constructed survival curves, as they related to the treatment group, using the Kaplan-Meier method, starting from the date of randomization in the control group and from the date of surgery in the tonsillectomy group. We tested differences between the groups using the log-rank test. We calculated the absolute difference and the $95 \%$ confidence intervals (CIs) in the proportions of recurrence between the groups at 5 months.

We determined the number of all episodes of pharyngitis, symptomatic days and absences from school or work per person-year using data obtained during follow-up. However, in the tonsillectomy group, we excluded from the risk time the individual recovery times immediately after tonsillectomy during which the patient had continuous throat pain (mean $17 \pm 6 \mathrm{~d}$ ). In scoring the Glasgow Benefit Inventory questionnaire, we averaged the responses to all 18 questions to give each question equal weight. We then transposed the average score onto a continual benefit scale ranging from -100 to 100 ; a score of -100 meant maximal harm, a score of 0 meant no change, and a score of 100 suggested maximal benefit to quality of life.

\section{Results}

\section{Participants and enrolment}

The first patient underwent randomization in October 2007, and the last patient completed the study in December 2010. A total of 260 candi- dates underwent screening, of whom we exluded 132; 42 candidates declined to participate (Figure 1). Most of the patients who were excluded had too few previous episodes of tonsillitis, had chronic tonsillitis or lived outside the study region. Of the 86 remaining patients, we randomly assigned 40 to the control group and 46 to the tonsillectomy group. We saw all of the patients at follow-up $(5.7 \pm 0.7$ mo for the control group, $6.2 \pm 0.5$ mo for the tonsillectomy group).

Almost all of the patients in the control group underwent surgery on their scheduled date; surgery was done before the limit of 5 months for 3 patients because of severe symptoms.

Two patients, one in each group, lost their symptom diaries but reported not having consulted a physician for throat pain. These patients were assumed to have had no symptoms during the study period. We found no clinically important differences in the baseline characteristics between the 2 groups (Table 1).

\section{Outcomes}

At 5 months, 1 patient in the control group and no patients in the tonsillectomy group had experienced an episode of severe pharyngitis (difference $3 \%, 95 \% \mathrm{CI}-2 \%$ to $7 \%$ ) (Table 2 and Appendix 2, available at www.cmaj.ca/lookup /suppl/doi:10.1503/cmaj.121852/-/DC1). Seventeen $(43 \%)$ patients in the control group and 2 (4\%) patients in the tonsillectomy group had consulted a physician for pharyngitis (difference $38 \%, 95 \%$ CI $22 \%$ to $55 \%) ; 32(80 \%)$ patients in the control group and $18(39 \%)$ patients in the tonsillectomy group had experienced an episode of acute pharyngitis (difference $41 \%, 95 \%$ CI $22 \%$ to $60 \%$ ) (Table 2 ).

During follow-up $(6.0 \pm 0.7 \mathrm{mo})$, the overall rate of pharyngitis and number of days with

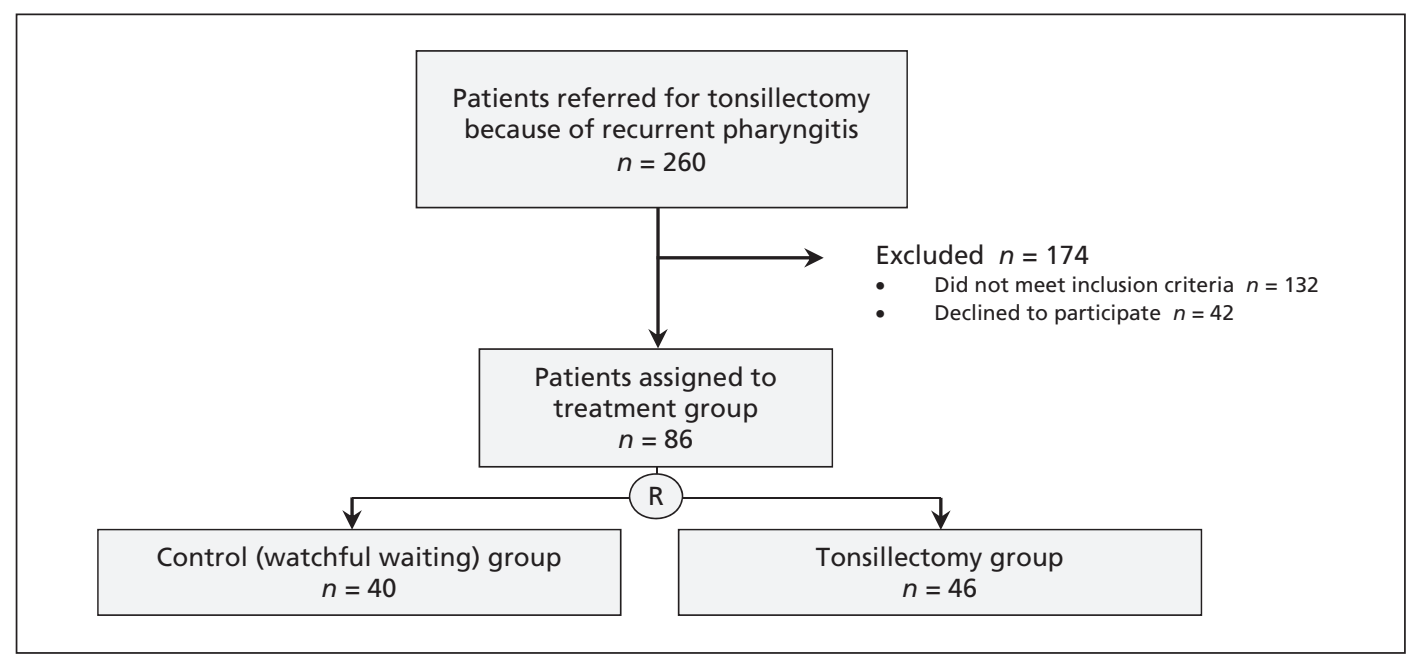

Figure 1: Enrolment of patients in the study. 
throat pain, fever, rhinitis and cough were significantly lower in the tonsillectomy group than in the control group (Table 3). Patients in both groups graded most of their throat pain as mild (Appendix 3, available at www.cmaj.ca/lookup /suppl/doi:10.1503/cmaj.121852/-/DC1). Patients in the tonsillectomy group also had significantly fewer days absent from school or work compared with patients in the control group. According to the postoperative quality-of-life questionnaire, patients in the tonsillectomy group were pleased with their surgeries (Glasgow Benefit Inventory overall score $27 \pm 12$, general health subscore $23 \pm 15$, social subscore $3 \pm 12$ and physical subscores $68 \pm 28$; Appendix 4 , available at www.cmaj.ca/lookup/suppl/doi:10 .1503/cmaj.121852/-/DC1).
The mean duration of postoperative throat pain was 17 days. Details of the surgeries and adverse effects are shown in Appendices 5, 6 and 7 (available at www.cmaj.ca/lookup/suppl/doi:10 .1503/cmaj.121852/-/DC1).

\section{Interpretation}

Adult patients with recurrent pharyngitis of any origin had very few severe episodes of pharyngitis (as determined by the presence of severe symptoms and serum levels of C-reactive protein), regardless of whether they underwent tonsillectomy. However, patients who did undergo surgery had fewer episodes of pharyngitis overall and less frequent throat pain than did patients in the control group. These reductions resulted in fewer medical visits and fewer absences from

Table 1: Demographic and baseline characteristics of adults with recurrent pharyngitis, by study group

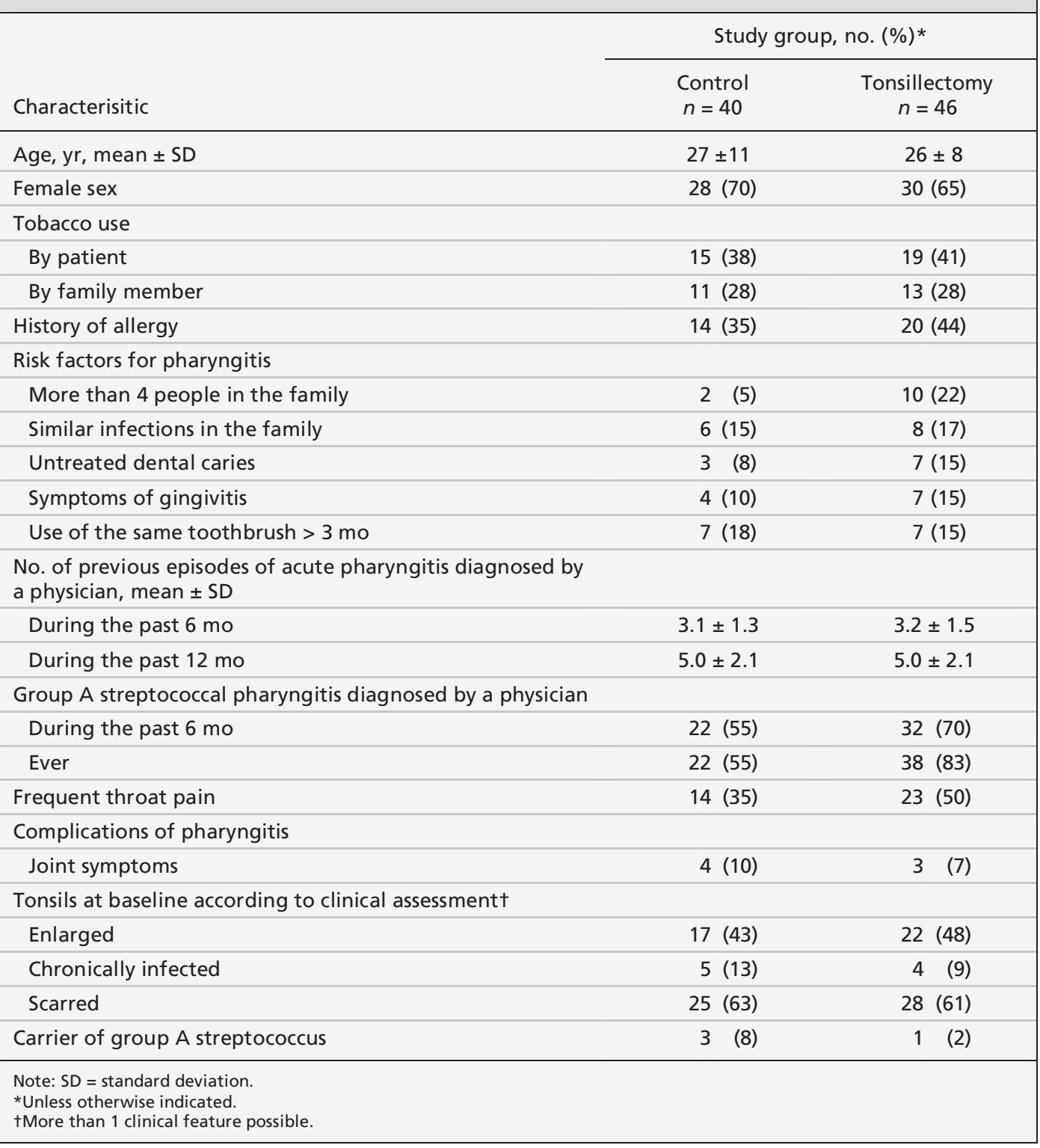


school or work. Patients who underwent surgery also felt that their quality of life improved. The most important morbidities related to surgery were postoperative throat pain and a small risk of postoperative bleeding.

The recent Cochrane review on tonsillectomy for recurrent tonsillitis found only a single trial involving adults, done by our research team, which included adults severely affected by recurrent group A streptococcal pharyngitis. ${ }^{2,3}$ In the current trial, we enrolled adults with recurrent pharyngitis of any origin, about half of whom had a streptococcal episode in the 6 months before randomization.

In both trials, patients showed similar benefit from tonsillectomy. In the previous trial, Alho and colleagues found an absolute difference of $30 \%$ in the proportion of patients who had pharyngitis with medical consultation and $25 \%$ in the proportion of patients who had pharyngitis without medical consultation between the surgical and control groups. ${ }^{3}$ In the current trial, these differences were $38 \%$ and $41 \%$, respectively. The results are remarkably similar, considering that the observation period was 5 months in the current trial, and only 3 months in the previous trial. Adult patients with recurrent pharyngitis seem to consult a physician similarly regardless of the cause of previous episodes.

Finally, the improved quality of life our patients reported after tonsillectomy is in agreement with the results of several previous studies. ${ }^{10-14}$

\section{Limitations}

Because we used an open trial design, the placebo effect may explain part of the subjective benefit after surgery, as suggested by the slight beneficial effect of tonsillectomy on cough and rhinitis.

The effect of surgery on pharyngeal symptoms was very good, and we believe this result cannot be due to expectation alone. Sham tonsillectomy has been suggested to control for this potential bias, ${ }^{15}$ but this suggestion seems naive. In our experience, patients know what the palatine tonsils are and describe their appearance when presenting with a sore throat. Thus, patients would undoubtedly know whether their tonsils had been removed, as would the physicians who assessed them during consultations for pharyngitis.

The wait-time for tonsillectomy is restricted by law to no more than 6 months, which resulted in a relatively short follow-up. However, we think that the short-term effect of tonsillectomy shows its overall usefulness. Moreover, considering the objective outcomes used in earlier trials, the effect of tonsillectomy does not depend on the length of follow-up. ${ }^{16}$ Any improvement in the pharyngitis rate in the control group during follow-up was likely due to the natural course of the disease. Thus it is unlikely that the patients in the control group reported negatively biased data in their diaries, and the difference between groups was likely due to the benefit of tonsillectomy rather than to any detrimental effect of remaining on the waiting list. Our use of waiting list controls also had advantages. With this

Table 2: Adult patients with recurrent pharyngitis who had an episode of acute pharyngitis within 5 months of enrolment in the study, by treatment group

\begin{tabular}{|c|c|c|c|}
\hline \multirow[b]{2}{*}{ Outcome } & \multicolumn{2}{|c|}{ Treatment group, no. (\%) } & \multirow[b]{2}{*}{$\begin{array}{l}\text { Difference, } \\
\%(95 \% \mathrm{Cl})\end{array}$} \\
\hline & $\begin{array}{c}\text { Control } \\
n=40\end{array}$ & $\begin{array}{c}\text { Tonsillectomy } \\
n=46\end{array}$ & \\
\hline \multicolumn{4}{|l|}{ Objective } \\
\hline Severe acute pharyngitis* & 1 (3) & $0 \quad(0)$ & $3(-2$ to 7$)$ \\
\hline $\begin{array}{l}\text { Any pharyngitis with } \\
\text { medical consultationt }\end{array}$ & $17(43)$ & 2 (4) & 38 (22 to 55$)$ \\
\hline \multicolumn{4}{|l|}{ Subjective } \\
\hline Any pharyngitis & $32(80)$ & 18 (39) & $41(22$ to 60$)$ \\
\hline \multicolumn{4}{|c|}{$\begin{array}{l}\text { Note: } \mathrm{Cl}=\text { confidence interval. } \\
\text { *Acute sore throat and signs suggesting the pharynx or tonsils as the origin of symptoms } \\
\text { (edema, erythema, exudative tonsillitis, anterior cervical lymphadenitis), in addition to serum } \\
\text { C-reactive protein level }>40 \mathrm{mg} / \mathrm{L} \text { either at the appointment or } 3 \text { days later. } \\
\text { †Acute sore throat and signs suggesting the pharynx or tonsils as the origin of symptoms } \\
\text { (edema, erythema, exudative tonsillitis, anterior cervical lymphadenitis). } \\
\text { fTwo consecutive days of throat pain recorded in symptom diary. }\end{array}$} \\
\hline
\end{tabular}

Table 3: Outcomes in adult patients with recurrent pharyngitis, by treatment group

\begin{tabular}{lccc} 
& \multicolumn{2}{c}{ Treatment group, ${ }^{*}$ mean \pm SD $\dagger$} \\
\cline { 2 - 3 } Outcome & Control & Tonsillectomy & $p$ \\
$n=40$ & $n=46$ & value
\end{tabular}

\section{Subjective}

\begin{tabular}{|lccc|}
\hline Episodes of sore throat, no. & $7.4 \pm 5.8$ & $1.5 \pm 2.4$ & $<0.01$ \\
\hline Throat pain, d & $40.2 \pm 60.5$ & $5.1 \pm 8.7$ & $<0.01$ \\
\hline Fever $\left(>37.5^{\circ} \mathrm{C}\right)$, d & $7.8 \pm 10.1$ & $2.2 \pm 5.2$ & $<0.01$ \\
\hline Rhinitis, d & $26.3 \pm 28.4$ & $10.2 \pm 14.3$ & $<0.01$ \\
\hline Cough, d & $16.0 \pm 17.7$ & $7.0 \pm 14.8$ & $<0.01$
\end{tabular}

\section{Objective}

Medical consultation for $1.0 \pm 1.4 \S$

$0.1 \pm 0.5$ $<0.01$

pharyngitis, no.

$\begin{array}{llll}\text { Absence from school or } & 6.6 \pm 11.8 & 3.3 \pm 10.0 & 0.02\end{array}$

work, d

Note: $\mathrm{SD}=$ standard deviation

*Mean length of follow-up was 5.7 mo for the control group and 6.2 mo for the

tonsillectomy group. Patients in the tonsillectomy group had $17( \pm 6) d$ of throat pain and 14

$( \pm 6) \mathrm{d}$ of absence from school or work during the immediate postoperative period. These

data are not included in the analysis.

tCalculated as events per year.

¥Mann-Whitney $U$ test.

§Only 1 episode was due to group A streptococci. 
method, the patients in the control group knew they would undergo surgery, and $67 \%$ of them agreed to participate. Only 3 patients in the control group asked to undergo surgery before the end of their 5-month wait, because of severe symptoms; no patients were lost to follow-up.

Our criterion for entry to the study was at least 3 disabling clinically significant episodes of pharyngitis per year. However, decisions to perform surgery with so few episodes were exceptions in which the episodes were very severe and prolonged; most patients had about 5 episodes during the previous year. This threshold for tonsillectomy is a bit lower than the criteria presented in certain guidelines. ${ }^{5}$ However, in our experience, these criteria are too strict for adult patients who opt for surgery earlier.

Based on the baseline characteristics of our patients and the high participation rate, we consider our results generalizable to the population of patients seen in otolaryngology outpatient clinics in Finland. ${ }^{17}$

\section{Conclusion}

Adult patients who had disabling pharyngitis involving the palatine tonsils more than 3 times per year that prevented normal functioning and led to medical consultation benefited from tonsillectomy. After tonsillectomy, patients had fewer episodes of pharyngitis and fewer days with sore throat, resulting in fewer medical visits and absences from school or work. However, pharyngitis and sore throats prevented by surgery were mild and probably of viral origin. The morbidity and complications related to tonsillectomy must be considered when physicians and patients decide whether the clinical benefits outweigh the risks of surgery. ${ }^{18}$

\section{References}

1. Bhattacharyya N, Kepnes LJ. Economic benefit of tonsillectomy in adults with chronic tonsillitis. Ann Otol Rhinol Laryngol 2002; 111:983-8.

2. Burton MJ, Glasziou PP. Tonsillectomy or adeno-tonsillectomy versus non-surgical treatment for chronic/recurrent acute tonsillitis. Cochrane Database Syst Rev 2009; (1):CD001802.

3. Alho OP, Koivunen P, Penna T, et al. Tonsillectomy versus watchful waiting in recurrent streptococcal pharyngitis in adults: randomised controlled trial. BMJ 2007;334:939.
4. Respiratory tract infections - antibiotic prescribing. London (UK): National Institute for Health and Clinical Excellence (NICE); 2008. Available: www.nice.org.uk/nicemedia/pdf/CG69 FullGuideline.pdf (accessed 2013 Jan. 20).

5. Management of sore throat and indications for tonsillectomy. Edinburgh (UK): Scottish Intercollegiate Guidelines network; 2010. Available: www.sign.ac.uk/pdf/sign117.pdf (accessed 2013 Jan. 20)

6. Bisno AL, Gerber MA, Gwaltney JM Jr, et al. Practice guidelines for the diagnosis and management of group A streptococcal pharyngitis. Infectious Society of America. Clin Infect Dis 2002; 35:113-25.

7. Robinson K, Gatehouse S, Browning GG. Measuring patient benefit from otorhinolaryngological surgery and therapy. Ann Otol Rhinol Laryngol 1996;105:415-22.

8. Wild D, Grove A, Martin M, et al. Principles of good practice for the translation and cultural adaptation process for patient reported outcomes (PRO) measures: report of the ISPOR task force for translation and cultural adaptation. Value Health 2005;8:94-104

9. Koo CY, Eisenhut M. Towards evidence-based emergency medicine: best BETs from the Manchester Royal Infirmary. Can inflammatory markers distinguish streptococcal from viral tonsillitis? Emerg Med J 2011;28:715-7.

10. Baumann I, Kucheida H, Bluemenstock G, et al. Benefit from tonsillectomy in adult patients with chronic tonsillitis. Eur Arch Otorhinolaryngol 2006;263:556-9.

11. Bhattacharyya N, Kepnes LJ, Shapiro J. Efficacy and quality-oflife impact of adult tonsillectomy. Arch Otolaryngol Head Neck Surg 2001;127:1347-50

12. Koskenkorva T, Koivunen P, Penna T, et al. Factors affecting quality-of-life impact of adult tonsillectomy. J Laryngol Otol 2009; 123:1010-4.

13. Richards AL, Bailey M, Hooper R, et al. Quality-of-life effect of tonsillectomy in a young adult group. ANZ J Surg 2007;77: 988-90.

14. Senska G, Ellermann S, Ernst S, et al. Recurrent tonsillitis in adults: quality of life after tonsillectomy. Dtsch Arztebl Int 2010; 107:622-8.

15. Crayford TJ. Recurrent pharyngo-tonsillitis. Time to stop doing tonsillectomies? BMJ 2007;334:1019.

16. van Staaji BK, van den Akker EH, van der Heijden GJ, et al. Adenotonsillectomy for upper respiratory infections: Evidence based? Arch Dis Child 2005;90:19-25.

17. Mattila PS, Tahkokallio O, Tarkkanen J, et al. Causes of tonsillar disease and frequency of tonsillectomy operations. Arch Otolaryngol Head Neck Surg 2001;127:37-44.

18. Randall DA, Hoffer ME. Complications of tonsillectomy and adenoidectomy. Otolaryngol Head Neck Surg 1998;118:61-8.

Affiliations: From the Department of Otorhinolaryngology (Koskenkorva, Koivunen, Kristo, Alho), Institute of Clinical Medicine, University of Oulu and Oulu University Hospital; the Department of Medical Microbiology (Koskela), Oulu University Hospital, Oulu, Finland; and the Medical Research Unit (Niemela), Seinajoki Central Hospital and University of Tampere, Tampere, Finland

Contributors: All of the authors contributed substantially to conception and design of the study. Timo Koskenkorva, Petri Koivunen, Aila Kristo and Olli-Pekka Alho collected and analyzed the data. All the authors drafted or revised the article for important intellectual content and approved the final version submitted for publication. 